\title{
Antifungal activity against Candida species and phenolic characterization of decoction, infusion and hydroalcoholic extract of cultivated Salvia officinalis $\mathbf{L}$.
}

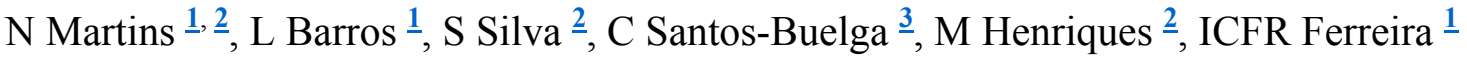 \\ - ${ }^{1}$ Mountain Research Centre (CIMO), ESA, Polytechnic Institute of Bragança, Portugal \\ - ${ }^{2}$ IBB - Institute for Biotechnology and Bioengineering, Centre of Biological Engineering, \\ University of Minho, Braga, Portugal \\ - ${ }^{3}$ GIP-USAL, Faculty of Pharmacy, University of Salamanca, Spain \\ - $\underline{\text { Congress Abstract }}$
}

The frequency and severity of diagnosed fungal infections is growing and, linked with this, it is observed an exacerbated increase of the antifungal drugs resistance. Candida albicans has been considered the main agent responsible for opportunistic pathogenic infections, but, recently, other non-C. albicans Candida species have also been considered [1]. In face to the increased number of Candida species drug resistant, it is crucial to use natural matrices to discover efficient alternative therapies to current antifungal agents. Salvia officinalis L., commonly known as sage, could be one of those natural matrices, containing a wide variety of bioactive molecules. Despite the existence of some reports on antimicrobial activity of sage, the most studied preparation is the essential oil, being scarce the aqueous extracts. The antifungal activity of decoction, infusion and hydroalcoholic extract of cultivated sage were evaluated, and further characterized in terms of phenolic composition (HPLC-DAD-ESI/MS). Antifungal activity was assessed against a total of 19 Candida strains of C. albicans, C. tropicalis, C. parapsilosis and C. glabrata recovered from different human biological samples. The most pronounced effect was observed against $C$. parapsilosis, followed by C. tropicalis and C. albicans. No effect was observed against the three C. glabrata strains. In general, decoction presented the most pronounced effect, followed by infusion and hydroalcoholic extract, varying directly according with the extract concentration. Decoction also showed the highest concentration in phenolic compounds, followed by hydroalcoholic extract and infusions. Rosmarinic acid and luteolin 7-O-glucuronide were the main phenolic compounds. Thus, the use of sage decoction, incorporated on daily food dietary, or even as complement to an antifungal therapy, could provide considerable benefits, not only by internal but also external use, without the common side effects reported for essential oil.

Keywords: Antifungal activity, Candida species, Salvia officinalis L., phenolic compounds References:

[1] Martins N, Ferreira ICFR, Barros L, Silva S, Henriques M. (2014). Mycopathol. DOI $10.1007 / \mathrm{s} 11046-014-9749-1$. 